\title{
Analysis of individual variations in the classical horizontal-vertical illusion
}

\author{
Kai Hamburger and Thorsten Hansen \\ Justus Liebig University Giessen, Giessen, Germany
}

\begin{abstract}
In the horizontal-vertical illusion (HVI), the length of the vertical line is overestimated, whereas in the bisection illusion (BI), the horizontal bisecting line is expected to be overestimated. Here, only half of our 22 observers showed the expected BI, whereas the other half underestimated the bisecting line. Observers also differed in their judgments of the strength of the HVI: The HVI was stronger for observers showing the classical bisection effect, and weaker or absent for those underestimating the bisecting line. To account for these results, we used a linear model to individually estimate the strength of two putative factors underlying both illusions. Whereas the strength of the HVI and BI were highly correlated, the estimated factors were uncorrelated. Therefore, in two control experiments, we then measured the pure horizontal-vertical (pHVI) and bisection ( $\mathrm{pBI}$ ) illusions. A significant correlation between the estimated factors and the measured illusion variants was found. Results were robust against variations of contrast, repetitive presentations, and choice of adjusted line. Thus, the classical HVI as an additive combination of two independent factors was confirmed, but we found considerable interindividual variations in the strength of the illusions. The results stress the importance of analyzing individual data rather than taking sample means for understanding these illusions.
\end{abstract}

In the classical horizontal-vertical illusion (HVI), the vertical line is generally perceived to be longer than a horizontal line of equal length. More than 150 years after the illusion of vertical overestimation was first scientifically studied by Fick $(1851,1852)$ and Oppel $(1855)$, this phenomenon and many others are still far from being fully understood (e.g., Westheimer, 2008). The understanding of this illusion is complex because of considerable interindividual differences in the strength and sometimes even the direction of the illusion, due to multiple factors that contribute to the illusion. The upside-down $\mathrm{T}$ figure commonly used to study the HVI contains more than an L-shaped figure - namely, an additional bisection effect, due to the overestimation of the dividing line (Ebbinghaus, 1913; Finger \& Spelt, 1947; Pan, 1934); this was first pointed out by Schumann (1900). Künnapas (1955) investigated these two independent illusions that contribute to the HVI - namely, the overestimation of the vertical line and the overestimation of the bisecting line. In the HVI, the two illusions work in the same direction and the overall illusion is the sum of both factors. In the bisection illusion (BI; T rotated by $90^{\circ}$ ), the two factors act in opposite directions, and the overall illusion is the difference between the two factors. Künnapas (1955) analyzed the observers' responses to the HVI and $\mathrm{BI}$ and reported that, on average, the pure bisection illusion ( $\mathrm{pBI}$ ) was considerably stronger $(9.6 \%)$ than the pure vertical illusion (pHVI; 3.42\%).

Another factor that complicates the understanding of the HVI is the existence of considerable interindividual differences between observers. Finger and Spelt (1947) used $\mathrm{T}$ and $\mathrm{L}$ figures to study the HVI. For all stimuli, they found an average overestimation of the vertical bar, but for each illusion, a subset of observers also underestimated the vertical bar. For example, the vertical bar was underestimated by 19 out of 72 observers of the upsidedown T stimulus (notably, the classical textbook example used to demonstrate the illusion) and by 20 observers of the $90^{\circ}$-rotated T. The number of observers who underestimated the vertical was even larger with the $\mathrm{L}$ figure (31 observers). An overview of studies of the HVI and its variants - the primary studies used in the present investigation-is given in Table 1. The HVI was first shown for the modality of vision, but it has also been investigated for the modalities of grasping and touch (for a review, see Gentaz \& Hatwell, 2004; Heller \& Joyner, 1993; Millar \& Al-Attar, 2000; Raudsepp \& Djupsjöbacka, 2005).

The main focus is on the additive and subtractive nature of the two factors (vertical overestimation/overestimation of the bisecting line), as suggested by Künnapas and others, some decades ago (see above). Will they prove to be adequate, or is there a need for revision of these assumptions? Therefore, we here reinvestigate different variations of the HVI. In the main experiment, we investigated the classical HVI and BI, and analyzed the data individually for each participant (in addition to the common group means). We found that only half of all participants showed the expected overestimation of the bisecting line in the BI. Then, we built a linear model to estimate the strength of

K. Hamburger, kai.hamburger@psychol.uni-giessen.de 
Table 1

Overview of Studies Investigating the Horizontal-Vertical Illusion and the Bisection Illusion

\begin{tabular}{|c|c|c|c|c|}
\hline Study & Procedure and Apparatus & $N$ & Stimulus & $\begin{array}{l}\text { Strength (Overestimation } \\
\text { of the Vertical) }\end{array}$ \\
\hline Avery \& Day (1969) & $\begin{array}{l}\text { Staircase procedure (light bars and } \\
\text { sliding shutters) }\end{array}$ & 14 & & $4.31 \%\left(0.29^{\circ}\right)$ \\
\hline \multirow[t]{4}{*}{ Finger \& Spelt (1947) } & $\begin{array}{l}\text { Visual adjustment (pulling cloth } \\
\text { tape to move steel tape as variable } \\
\text { part of the stimulus) }\end{array}$ & 72 & & $7.2 \%\left(0.65^{\circ}\right)$ \\
\hline & & & & $3.2 \%\left(0.29^{\circ}\right)$ \\
\hline & & & & $1.0 \%\left(0.09^{\circ}\right)$ \\
\hline & & & & $8.5 \%\left(0.77^{\circ}\right)$ \\
\hline $\begin{array}{l}\text { Hamburger, Hansen, \& } \\
\text { Gegenfurtner (2007) }\end{array}$ & $\begin{array}{l}\text { Visual adjustment (keypresses } \\
\text { change stimulus on monitor) }\end{array}$ & 20 & & $6.66 \%\left(0.64^{\circ}\right)$ \\
\hline \multirow[t]{4}{*}{ Present study } & $\begin{array}{l}\text { Visual adjustment (keypresses } \\
\text { change stimulus on monitor) }\end{array}$ & 22 & & $6.46 \%\left(0.68^{\circ}\right)$ \\
\hline & & & & $-6.17 \%$ to $+9.17 \%\left(-0.65^{\circ}\right.$ to $\left.+0.96^{\circ}\right)$ \\
\hline & & & & $2.28 \%\left(0.24^{\circ}\right)$ \\
\hline & & & & $-7.54 \%$ to $+6.17 \%\left(-0.79^{\circ}\right.$ to $\left.+0.65^{\circ}\right)$ \\
\hline \multirow[t]{2}{*}{ Künnapas (1955) } & $\begin{array}{l}\text { Method of constant stimuli, ink on } \\
\text { cardboard }\end{array}$ & 10 & & $13.02 \%\left(0.37^{\circ}\right)$ \\
\hline & & & & $6.18 \%\left(0.18^{\circ}\right)$ \\
\hline Gardner \& Long (1960a) & $\begin{array}{l}\text { Visual adjustment (turning knob to } \\
\text { move cardboard) }\end{array}$ & 128 & & $\begin{array}{l}\text { adjust horizontal: } 28.28 \%\left(1.06^{\circ}\right) \\
\text { adjust vertical: } 11.91 \%\left(0.45^{\circ}\right)\end{array}$ \\
\hline
\end{tabular}

the two pure illusions (misjudgment of the bisecting line and overestimation of the vertical) for each participant, based on the idea that these pure illusions combine additively in the HVI and subtractively in the BI (Künnapas, 1955). The basic linear formulas could be confirmed, but we found that different observers either over- or underestimated the bisecting line. In two control experiments, we found that the individual strengths of the two pure illusions as estimated by the model are highly correlated with the measured observers' misjudgments in variants of these illusions. Additionally, different parameters of the presentation and procedure were varied to rule out that the illusions are influenced by stimulus contrast, orientation of the line to be adjusted, or repetitive presentations.

\section{EXPERIMENT}

\section{Method}

Stimuli. Here, we reexamined the HVI with a purely visual technique, whereby observers adjusted a test line presented on a computer monitor to the point of subjective equality (the same procedure used in Hamburger, Hansen, \& Gegenfurtner, 2007). Two stimuli were investigated in the main experiment: an HVI consisting of an upside-down T (Figure 1A), and a BI made of the HVI stimulus rotated clockwise by $90^{\circ}$ (Figure 1B). Stimuli were created with the Psychophysics Toolbox (Brainard, 1997; Pelli, 1997) in
MATLAB (The MathWorks, Natick, MA) and were presented in two luminance contrast conditions, a medium-contrast condition with a Weber contrast of $67 \%$ and a low-contrast condition with a Weber contrast of $18 \%$. In the medium-contrast condition, the luminances of the background and the lines were $78.2 \mathrm{~cd} / \mathrm{m}^{2}$ and $25.80 \mathrm{~cd} / \mathrm{m}^{2}$, respectively, and $56.76 \mathrm{~cd} / \mathrm{m}^{2}$ and $46.44 \mathrm{~cd} / \mathrm{m}^{2}$ in the low-contrast condition. Stimuli subtended $17^{\circ}$ of the visual field. The length of the horizontal and vertical lines was $10.4^{\circ}(300$ pixels $)$ at the point of physical equality. Lines were $0.14^{\circ}$ (4 pixels) wide. The initial length of the bisecting line to be adjusted randomly varied in each trial between -40 pixels (enhancement of the illusion) and +80 pixels (compensation of the illusion). The fact that the length of the adjustable line in the initial presentation differed in each trial ensured that participants could not simply replicate the number of keypresses once the stimulus was shown again.

Participants. Twenty-three naive student participants (11 females, 12 males) from the University of Giessen, with normal or corrected-to-normal visual acuity, took part. Their ages ranged between 20 and 48 years. One participant was excluded from the study because she reported problems doing the adjustment task.

Task and Procedure. Participants adjusted the length of the vertical line (in the HVI) or the length of the horizontal line (in the BI) by pressing the right and left arrow keys of a keyboard to the point of subjective equality. Coarse adjustments were made by holding down a key, whereas fine adjustments were made by pressing a key only once. Observers were instructed to adjust the point of subjective equality (length) from two directions (ascending and descending). Possible adjustments ranged from $-1.4^{\circ}$ ( -40 pixels $)$ - that is, a physical enhancement of the expected illusory effect- to $+2.8^{\circ}$ 
A

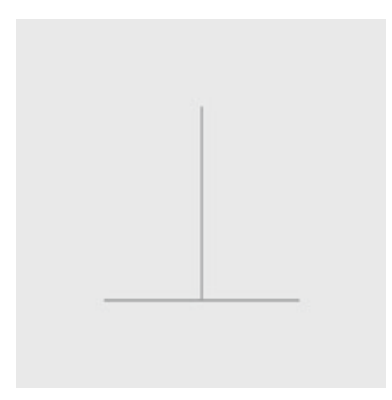

C

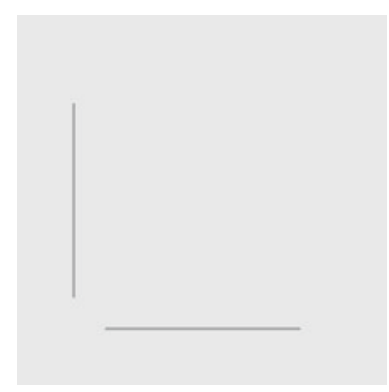

B

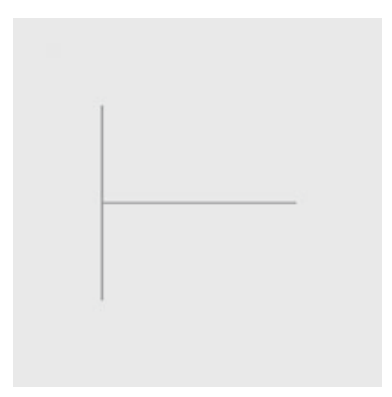

D

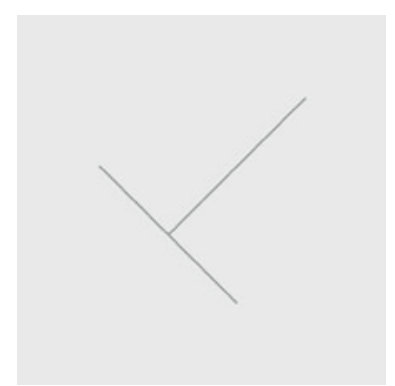

Figure 1. Stimuli used in the experiment: (A) Horizontalvertical illusion (HVI). (B) Bisection illusion (BI). (C) L stimulus to investigate the pure horizontal-vertical illusion (pHVI). (D) Stimulus to investigate the pure bisection illusion (pBI).

( +80 pixels $)$ - that is, a compensation of the expected illusory effect. When participants reached the point where they perceived the lines to be equal in length, they had to press the space bar to indicate their judgment and to start the next trial. After each trial the screen was blank (neutral gray) for $1 \mathrm{sec}$.

Observation was binocular and target stimuli were presented in the center of the monitor at the participants' line of sight. Stimuli were presented four times in randomized order. The initial length of the adjustable line was randomly varied in each trial. Stimuli were presented on a 21-in. Iiyama Vision Master Pro 513 CRT monitor with a resolution of $1,154 \times 768$ pixels that was driven by an NVIDIA Quadro NVS 285 graphics card at a refresh rate of $85 \mathrm{~Hz}$ noninterlaced. The experiment was conducted in an otherwise dark room. Observation distance was $60 \mathrm{~cm}$. A chinrest was used for head stabilization, but participants were free to move their eyes. Free viewing yields a robust estimate of the illusion because the illusions have been reported to be greater with free viewing (Künnapas, 1958) and they do not differ depending on the point of fixation (Piaget, Matalon, \& Bang, 1961). No time limit was given, but participants normally completed a single trial within $1 \mathrm{~min}$.

\section{Results}

The two contrast conditions revealed identical results. Therefore, these two conditions were merged in the following analysis. In the HVI, almost all participants (19 of 22) perceived the vertical line to be longer (Figure $2 \mathrm{~A}$ ). The average overestimation was $6.46 \%$ or $0.68^{\circ}$. Only 3 participants had a nominally negative HVI, which was very small $\left(0.31 \% \pm 0.24 \% S E M\right.$, or $\left.0.03^{\circ} \pm 0.03^{\circ}\right)$. In the BI, only half of all observers showed the expected overestimation of the bisecting line, whereas the other half perceived the vertical, bisected line to be longer (Fig- ure $2 \mathrm{~A})$. The strength of the HVI ranged from $-0.58 \%$ to $+13.08 \%$ (or $-0.06^{\circ}$ to $1.37^{\circ}$ ) and the strength of the BI ranged from $-6.17 \%$ to $+9.17 \%$ (or $-0.65^{\circ}$ to $0.96^{\circ}$ ). Thus, the absolute range of the strength of both illusions was quite similar (for HVI, $13.66 \%$ or $1.43^{\circ}$, and for BI, $15.33 \%$ or $1.61^{\circ}$ ). The strengths of the two illusions were significantly correlated ( $r=.81, p<.001$; Figure $2 \mathrm{~B})$. Note that considering only sample means would indicate a strong HVI $\left(6.46 \%\right.$ or $\left.0.68^{\circ}\right)$, and a weak and almost absent BI $\left(0.35 \%\right.$ or $\left.0.04^{\circ}\right)$. Taking the mean is problematic for the BI because it hides strong individual illusions in different directions, depending on the participant.

Künnapas (1955) proposed an additive combination of two illusions that determine the overall strength of the HVI and the BI. These two individual illusions are an overestimation of the bisecting line $I_{\text {bisect }}>0$ and an overestimation of the vertical line $I_{\text {vertical }}>0$. For the HVI, both individual illusions work in the same direction, so that the overall strength of the illusion is the sum of the individual illusions:

$$
I_{\mathrm{HVI}}=I_{\text {bisect }}+I_{\text {vertical }} \text {. }
$$

For the BI, both illusions work in different directions, so that the overall strength of the illusion is the difference of the individual illusions:

$$
I_{\mathrm{BI}}=I_{\text {bisect }}-I_{\text {vertical }} \text {. }
$$

Künnapas (1955) reported that the overestimation of the bisecting line $I_{\text {bisect }}$ was on average $9.6 \%$ and much stronger than the average overestimation of $3.42 \%$ of the vertical line $I_{\text {vertical }}$.

Our data show that these formulas need to be evaluated individually for each participant. Most important, half of the participants in the present study showed a negative $\mathrm{BI}$ - that is, an underestimation of the vertical. This is in accordance with the above equations only if one allows negative values for the illusory estimation of the bisecting line - that is, $I_{\text {bisect }}<0$.

Next we determined the individual values $I_{\text {bisect }}$ and $I_{\text {vertical }}$ for each observer by solving the overdetermined system of linear equations given by the following 16 equations ( 2 stimuli $\times 2$ contrast conditions $\times 4$ presentations).

$$
\begin{aligned}
& I_{\mathrm{HVI}}(\text { presentation } 1, \text { condition } 1)=I_{\text {bisect }}+I_{\text {vertical }} \\
& I_{\mathrm{HVI}}(\text { presentation } 2, \text { condition } 1)=I_{\text {bisect }}+I_{\text {vertical }} \\
& I_{\mathrm{HVI}}(\text { presentation } 3, \text { condition } 1)=I_{\text {bisect }}+I_{\text {vertical }} \\
& I_{\mathrm{HVI}}(\text { presentation } 4, \text { condition } 1)=I_{\text {bisect }}+I_{\text {vertical }} \\
& I_{\mathrm{HVI}}(\text { presentation } 1, \text { condition } 2)=I_{\text {bisect }}+I_{\text {vertical }} \\
& I_{\mathrm{HVI}}(\text { presentation } 2, \text { condition } 2)=I_{\text {bisect }}+I_{\text {vertical }}
\end{aligned}
$$

$I_{\mathrm{HVI}}\left(\right.$ presentation 4, condition 2) $=I_{\text {bisect }}+I_{\text {vertical }}$ $I_{\mathrm{BI}}($ presentation 1, condition 1$)=I_{\text {bisect }}-I_{\text {vertical }}$ $I_{\mathrm{BI}}($ presentation 1, condition 2$)=I_{\text {bisect }}-I_{\text {vertical }}$

\section{$I_{\mathrm{BI}}($ presentation 4, condition 2$)=I_{\text {bisect }}-I_{\text {vertical }}$}

The ordinary least squares solution to the linear system of equations was determined using the lscov function of 
A

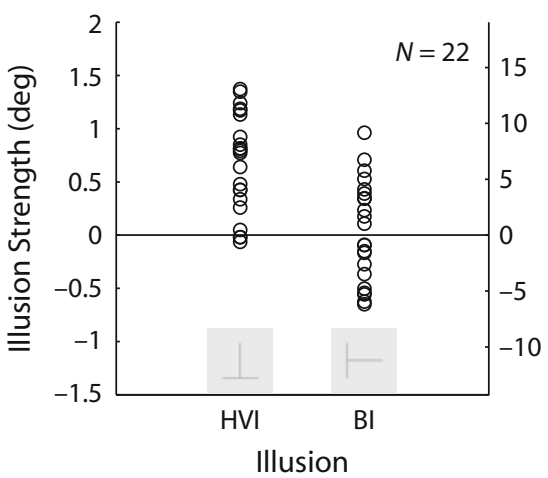

B

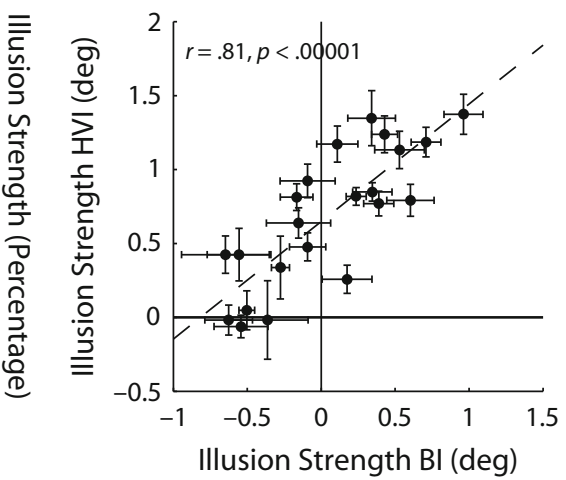

C

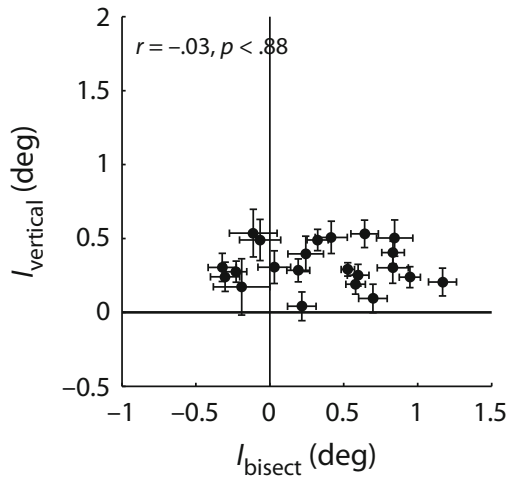

Figure 2. Results for the horizontal-vertical illusion (HVI) and the bisection illusion (BI). (A) Individual strengths of illusory effects in the HVI and BI $(N=22)$ are significantly correlated. (B) Error bars indicate the standard error of the mean (SEM). (C) Estimation of the individual strengths of the two factors $\left(I_{\text {vertical }}\right.$ and $\left.I_{\text {bisect }}\right)$ that contribute to the illusion. These two factors of pure illusions are uncorrelated. Error bars indicate the estimated standard error from the least squares solution.

MATLAB. The linear system of equations was solvable with small mean squared error for all participants (SEM ranging between 0.1 and 2.0, median 0.2 ), showing that the basic idea of a linear combination of individual illusions as proposed by Künnapas is valid.

The distribution of the two factors $I_{\text {vertical }}$ and $I_{\text {bisect }}$ for the 22 participants is shown in Figure 2C. The data reveal a considerable variation of these factors across participants. The overestimation of the vertical $I_{\text {vertical }}$, as determined by the model, varied between $0.40 \%$ and $5.10 \%$ (equivalent to $0.04^{\circ}$ and $0.54^{\circ}$ ), whereas the overestimation of the bisecting line showed a considerably larger variation between $-3.06 \%$ and $11.13 \%$ (equivalent to $-0.32^{\circ}$ and $1.17^{\circ}$ ). The absolute range of the two estimated illusions differed considerably: The range was rather small for $I_{\text {vertical }}\left(4.71 \%\right.$ or $\left.0.49^{\circ}\right)$, and larger for $I_{\text {bisect }}\left(14.19 \%\right.$ or $\left.1.17^{\circ}\right)$.

Here, we found further evidence for two factors in the HVI, as suggested by Finger and Spelt (1947) and Künnapas (1955). However, we found considerable interobserver variations in the estimated strength of the pure illusions $I_{\text {vertical }}$ and in particular of $I_{\text {bisect }}$, which can be negative for some participants. The additive equations can only account for the data if the pure illusions $I_{\text {vertical }}$ and $I_{\text {bisect }}$ are determined individually for each participant and if one allows negative values for $I_{\text {bisect }}$.

Next, we aimed at directly measuring the two pure illusions to compare them with the model predictions. Therefore, we used two new stimuli, an L figure (pHVI; Figure $1 \mathrm{C}$ ), to investigate the pHVI without the potential contributions of a bisection illusion (Control Experiment 1 ); and a $45^{\circ}$-rotated T (pBI; Figure $\left.1 \mathrm{D}\right)$ to investigate the $\mathrm{pBI}$ without the potential contributions of the HVI (Control Experiment 2).

\section{CONTROL EXPERIMENTS}

We ran two control experiments to compare the estimated factors with the strength of the pure illusion.

\section{Control Experiment 1 Pure Horizontal-Vertical Illusion (pHVI)}

To separate the vertical and bisection effects from each other and to measure the strength of the pure vertical effect, we conducted a control experiment in which we used an $\mathrm{L}$ figure made of a horizontal and a vertical bar that were nonadjacent to each other (pHVI; Figure 1C).

\section{Method}

Stimuli. We used an L stimulus made of a vertical line and a horizontal line that did not touch or bisect each other (pHVI; Figure 1C). Stimulus dimensions were identical to the main experiment. This stimulus is similar to the one used by Finger and Spelt (1947; their Figure 1A), but in their study only the vertical line was to be adjusted, and for adjustment of the horizontal line the image board had to be rotated by $90^{\circ}$. We used identical stimuli with which observers could adjust either the horizontal or the vertical line in different trials.

Participants. A subset of 6 naive students ( 3 females, 3 males) who also participated in the main experiment took part in the control experiment. The participants had normal or corrected-to-normal visual acuity and were between 20 and 38 years of age.

Procedure. The task and procedure were identical to those in the main experiment. Either the horizontal or the vertical line could be adjusted in length. Sixteen trials were run: In half of the trials, the horizontal line had to be adjusted until it was perceived to be equally as long as the vertical line, and in the other half, the length of the vertical line was adjusted until it perceptually matched the horizontal line. Adjustments changed the vertical line at the upper end and the horizontal line at the right end to ensure that both lines never touched or intersected.

\section{Results}

No matter which line was manipulated, all observers overestimated the length of the vertical line (Figure 3A; $\mathrm{pHVI})$. The strength of the illusions ranged between $1.00 \%$ and $+4.58 \%$ (or $0.11^{\circ}$ and $0.48^{\circ}$ ), with an absolute range of $3.58 \%$ or $0.38^{\circ}$ and a mean of $2.28 \% \pm 1.37 \%$ SEM (or $0.24^{\circ} \pm 0.14^{\circ}$ ). The two manipulations (adjustment of either the horizontal or the vertical line) did not differ significantly within this group. However, descriptively, the HVI was slightly stronger if the vertical line was adjusted 
A

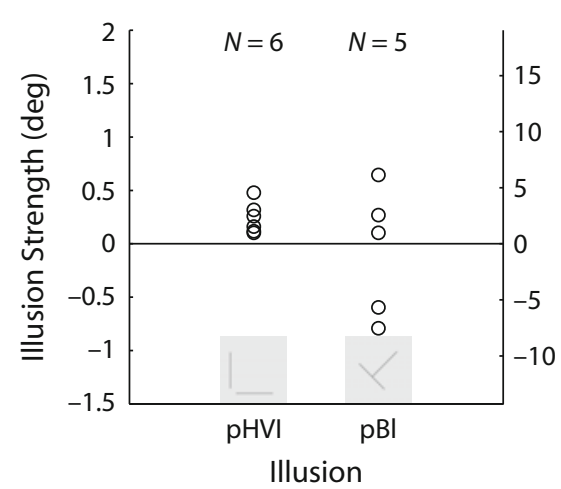

B

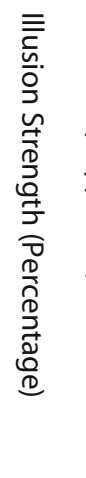

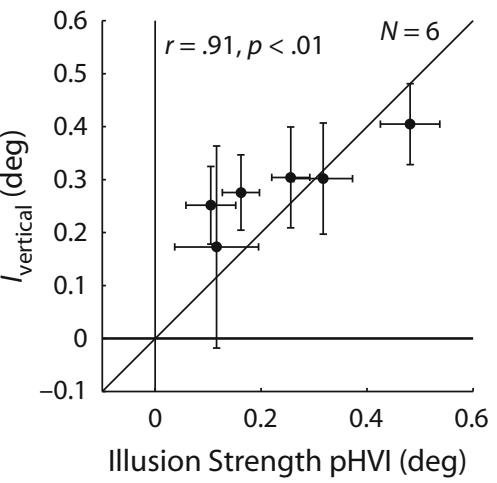

C

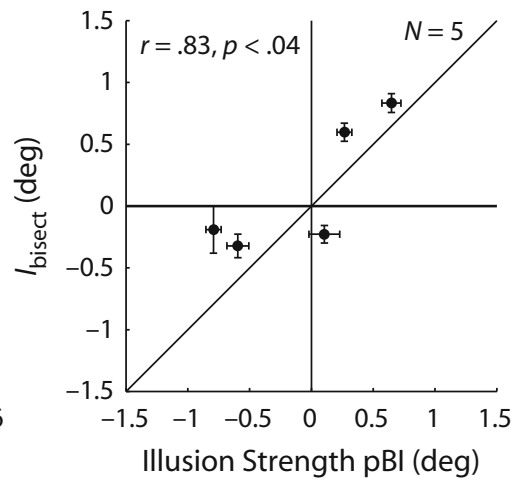

Figure 3. Results for the pure illusions pHVI and pBI in which the vertical overestimation and bisection effect were investigated independently of each other. (A) Individual strength of illusory effects in the pHVI $(N=6)$ and pBI $(N=5)$. We found a high correlation between the factors estimated by the model and the measured illusions both for $(B)$ the overestimation of the vertical $(r=$ $.91, p<.01)$ and $(C)$ the overestimation of the bisecting line $(r=.83, p<.04)$.

than if the horizontal line was adjusted. But this difference turned out to be insignificant. More important, we found a high correlation between the strength of the measured pHVI and the estimated strength of the pure vertical effect $I_{\text {vertical }}$ (Figure 3B, $r=.91, p<.01$ ).

\section{Discussion}

Everything from the previous experiment was kept constant, except that this time the two lines did not touch or bisect each other. With this control paradigm, we could show that the pure vertical illusion is rather small $(2.28 \%)$ in comparison with the classical HVI (mean 6.46\%). Furthermore, the vertical underestimation only occurs if the horizontal line bisects the vertical line. The vertical overestimation seems to be a general effect, being present in all participants with little variation. This suggests that the large individual variations we measured in the main experiment for the HVI and the BI are due to individual variations in the pure bisecting effect. The illusion is not diminished by previous exposition to the similar illusions (HVI and BI), since naive and experienced observers show similar results. We did not find evidence for an overestimation of the standard line that has been reported to increase the HVI if the vertical line is the standard (Gardner \& Long, 1960a, 1960b).

\section{Control Experiment 2 Pure Bisection Illusion (pBI)}

To separate the vertical and bisection effects from each other and to measure the strength of the pure bisection effect, we conducted a second control experiment in which we tilted the inverted $\mathrm{T}$ by $45^{\circ}$ to the right ( $\mathrm{pBI}$; Figure 1D).

\section{Method}

Stimuli. We used a T tilted by $45^{\circ}$ as the stimulus (Figure 1D). The downward-pointing diagonal was fixed and the upward-pointing bisecting line was adjustable. In this stimulus, no vertical elements were present, so the classical vertical effect can be assumed to be absent (since the two vertical effects of the rotated lines cancel each other), and only the pure bisection effect is present.
Participants. Five students ( 2 females, 3 males), who also participated in the main experiment and had normal or corrected-tonormal visual acuity, took part. Their ages ranged between 20 and 38 years.

Procedure. The task and procedure were identical to those used in the main experiment and Control Experiment 1. The bisecting line could be adjusted by changing its lengths at the top right end. Each participant ran a total of eight trials, with breaks of $5 \mathrm{sec}$ between the trials.

\section{Results}

Three participants showed an overestimation of the bisecting line, whereas 2 participants showed an underestimation of the bisecting line (Figure 3A; pBI). The strength of the illusions ranged between $-7.54 \%$ and $6.17 \%$ (or $-0.79^{\circ}$ and $0.65^{\circ}$ ), with an absolute range of $13.71 \%$ $\left(1.44^{\circ}\right)$. Those participants with a negative $\mathrm{pBI}$ also had a negative BI in the main experiment - that is, they underestimated the horizontal bisecting line. More important, we found a high correlation between the strength of the measured illusion $\mathrm{pBI}$ and the estimated strength of the pure vertical effect $I_{\text {bisect }}$ (Figure $3 \mathrm{C}, r=.83, p<.04$ ).

\section{Discussion}

Here, we investigated the bisection effect in the absence of the classical vertical illusion. Two out of 5 participants underestimated the bisecting line, whereas the others showed the classical overestimation of the bisecting line. We found that the bisection effect greatly varies across participants and can result in either an over- or underestimation of the bisecting line. Our model is supported by these empirical data, since all values are closely distributed around the ideal position at the main diagonal.

\section{GENERAL DISCUSSION}

\section{Summary of Results}

We replicated the overestimation of the vertical in the classical HVI as reported previously with an upside-down T stimulus (Avery \& Day, 1969; Brosvic \& Cohen, 1988; Finger \& Spelt, 1947; Künnapas, 1955; McBride, Risser, 
\& Slotnick, 1987). However, when this stimulus is rotated by $90^{\circ}$ to create a bisection illusion, we found that only half of the observers showed the expected overestimation of the bisecting line.

We confirmed previous findings suggesting that two different factors contribute to the HVI: misjudgments of the vertical line and the bisecting line. The vertical line was always overestimated and the bisecting line could be either over- or underestimated. We estimated the size of these individual illusions from the responses in the HVI and BI using a linear model, and found a high correlation between the estimated values and the strengths of the illusions measured in two control experiments.

We further demonstrated that the vertical is always overestimated if the stimulus components do not touch or bisect each other (Control Experiment 1), and that a bisecting line can be either over- or underestimated even if there are no vertical or horizontal components in the pure bisection illusion (Control Experiment 2). The results varied greatly between participants, and the classical equations for describing the effects only account for the data if the bisecting line could be either over- or underestimated. Our data show that a pure vertical illusion occurs with similar strengths for almost all observers (model prediction $I_{\text {vertical }}$ in the main experiment and measured pHVI in Control Experiment 1), whereas the pure bisecting illusion (model prediction $I_{\text {bisect }}$ in the main experiment and measured $\mathrm{pBI}$ in Control Experiment 2) differs considerably and can also result in an underestimation of the bisecting line. The same holds if totally inexperienced participants are tested with the two control experiments (the data of these naive participants are not reported in detail here; Control Experiment 1, $n=10$; Control Experiment 2, $n=8$ ).

Furthermore, most previous studies investigated the HVI and BI that include two contributing factors (vertical overestimation and misjudgment of the bisecting line) rather than investigating stimuli that separate both factors. Here, we not only controlled for the two different factors, but we used a linear model to predict the strengths of the illusions in two control experiments for each participant from the measured illusions in HVI and BI.

\section{Comparison With Previous Studies}

The average illusory effect of $6.46 \%$ in the HVI when measured with the classical upside-down $\mathrm{T}$ stimulus was similar to results from previous studies (7.2\%, Finger \& Spelt, 1947; 6.66\%, Hamburger et al., 2007; 13.02\%, Künnapas, 1955). A considerably larger value of $28.28 \%$ was reported by Gardner and Long (1960a). They used a setup in which white lines appeared in complete darkness, so that only the stimulus was visible. The lack of any remote surrounding frames that could be used for reference may account for the high value. Without any reference frame, the participants are not limited in their compensation of the illusion by any remote boundaries. Only one study reported a negative effect in the classical HVI, such that the vertical was perceived as shorter (Finger \& Spelt, 1947). They found that the vertical was underestimated by 19 out of 72 participants. We found only a few (3 out of 22) observers who showed a tiny underestimation of $0.31 \%$ in comparison with the large overestimation of $7.53 \%$ (averaged for 19 out of 22 observers). We interpret these results to mean that the classical HVI is a very robust effect that can be found in almost every participant.

Investigating the pHVI using L-shaped figures, we found a considerably smaller illusion (2.28\%). This is in accordance with Künnapas (1955), who reported an average size of $3.51 \%$ for L-shaped figures. The stronger illusion in the classical upside-down T version of the HVI can be attributed to the additional overestimation of the bisecting line.

We showed that the bisecting line can be over- or underestimated. This finding is in agreement with Finger and Spelt (1947), who reported that 20 out of 72 observers underestimated the vertical in the BI. They only stated how many participants did not confirm their hypotheses. Here, we explicitly analyzed the interindividual differences. Other studies have reported only sample means (Künnapas, 1955) and found a classical overestimation of the bisecting line by $6.18 \%$ for the 10 observers studied. Ten observers is a rather small number, suggesting that only observers who overestimated the bisecting line may have been included in the study by Künnapas (1955).

Besides individual differences, a general reason for some ambiguity of the results in the literature on HVI and its variants may be due to differences in the method of collecting the data and in different apparatuses used, as summarized in Table 1.

\section{Possible Explanations for the Illusory Misjudgments}

Avery and Day (1969) found that the misjudgment depends on the orientation of the line relative to the retinal image: With upright viewing, the vertical is overestimated, whereas with recumbent viewing, the horizontal is overestimated. Since the monocular visual field is less asymmetric than the combined visual field, this theory predicts that the illusion should be reduced with monocular presentation. Indeed, the illusion was reliably reduced with monocular presentation under conditions that affected the asymmetry of the phenomenal visual field (Prinzmetal \& Gettleman, 1993). Another influential explanation of the HVI was put forward by the Gestalt psychologist Kurt Koffka (1935/1999). He proposed that visual phenomenal space is not a one-to-one mapping of the physical space, but is distorted or anisotropic even in the cardinal directions. Along similar lines, it has been argued that the visual field is elongated in the horizontal direction, and that the vertical-horizontal illusion is a kind of framing effect (Künnapas, 1957, 1959). In a similar way, Westheimer (2008, p. 2134) argues that "there may even be simpler underlying principles, such as a vertical/horizontal anisotropy or one of oblique compared to cardinal orientation of contours."

Brosvic and Cohen (1988) reported that the vertical illusion also exists in the absence of a horizontal comparison and can be accounted for by an overestimation in the vertical plane. Overestimation of vertical elements 
also occurs in geometric objects observed in their natural and perceptually rich environment (Chapanis \& Mankin, 1967). For example, the Gateway Arch, a $690 \times 690 \mathrm{ft}$ steel building in St. Louis, Missouri, having no line bisection or vertical line, is perceived as being taller than it is wide. It has also been reported that the context of the surround greatly influences the perceived line length in the HVI (Armstrong \& Marks, 1997). Westheimer (2008) suggested that geometrical-optical illusions arise in the transformation from physical to visual space, and can be modeled by an affine transformation.

A possible explanation based on an asymmetry of the eye movement system has already been ruled out (Gregory, 1968). Gregory argued that the explanation for these length distortions cannot be found in the eyes but rather in the brain. He distinguished between optical, sensory, and perceptual illusions (Gregory, 1968), with geometricoptical illusions subsumed under the term "perceptual illusions." Top-down processes such as conceptual or perceptual knowledge and "sideways" (rules) are crucial in perceptual illusions (Gregory, 1997a, 1997b). Corticocortical and corticothalamic feedback connections are common at the higher stages of visual processing and may form the neural substrate of the top-down processes (Felleman \& van Essen, 1991; Rockland \& Pandya, 1979; Sillito, 1995; van Essen, 1985). To Gregory (2001), “perceptions are guesses - predictive hypotheses - of what may be out there" in the world (p. 21). In illusions, especially in geometric-optical illusions, these personal hypotheses may be wrong if based on false assumptions and may therefore lead to illusory effects of individually different qualities. In the bisection illusion, observers may overestimate the bisecting line because they tend to compare its length with the length of the individual bisected lines, or observers may underestimate the bisecting line because they tend to compare a single item, the bisecting line, with two bisected lines.

However, such attempts for explaining the HVI are beyond the scope of the present work.

\section{Evidence in the Haptic Domain}

So far, we have almost exclusively concentrated on the HVI and BI in the visual domain. Taking results from other domains, such as haptics, into account can give valuable insights into the nature of these two illusions. If the same factors influence the strength of the illusion in both domains, this indicates similar perceptual processes underlying the illusion. It could be shown that bisection effects and vertical overestimation are also present when participants judge the length of the elements by touch (Heller, Calcaterra, Burson, \& Green, 1997; Heller \& Joyner, 1993). Heller and colleagues found that the HVI occurs with touch but that the magnitude of the effect depends on visual experience and stimulus size. Heller and Joyner speculated that a possible explanation for these illusions might be an elongated haptic field analogous to the elongated visual field as described above. They "describe[d] the haptic field as oval, in a similar way, since one's horizontal space (between laterally outstretched hands, shoul- der height; frontoparallel plane) is larger than one's frontal (or vertical) space" (Heller \& Joyner, 1993, p. 426). They did not just point to possible similarities between sight and touch but also assumed that a "history of input from more than one modality may lead to increased accuracy in perception" (Heller \& Joyner, 1993, p. 427). Heller and Joyner presented their stimuli flat on a table, where exploratory movements are radial for vertical segments and tangential for horizontal segments. When $\mathrm{T}$ and $\mathrm{L}$ figures were presented in a frontoparallel plane, where all exploratory movements are tangential, the bisection effect in the $\mathrm{T}$ figure remained, whereas the vertical segments in the L figure were no longer overestimated (Day \& Avery, 1970; Deregowski \& Ellis, 1972). These works point out the importance of scanning (exploration) methods, spatial reference cues or reference frames, anchors, and so on (for a review, see Gentaz \& Hatwell, 2004; Heller \& Joyner, 1993; Millar \& Al-Attar, 2000). The strength of the illusion was generally found to be similar for sighted and blind participants, ruling out that visual imagery or visual representations are responsible for the haptic illusion.

Taken together, the HVI is due to a common perceptual illusion that operates in both vision and haptics - namely, bisection - and due to processes specific to each modality - namely, anisotropy of the visual field and overestimation of radial versus tangential manual exploratory movements (Gentaz \& Hatwell, 2004). Therefore, it will be of interest to investigate the visual effects found and modeled in the present study in the domain of haptics. More precisely, if a common perceptual illusion underlies the bisection effect, the strong interindividual differences in the visual bisection illusion should also be present in the same magnitude and direction in the haptic domain. Overall, purely visual explanations may be incomplete because higher level processes common to different modalities can be involved.

\section{Summary}

We found that two components are present in the classical HVI: an overestimation of the vertical and a misjudgment of the bisecting line. The bisecting line can be either over- or underestimated. We estimated these two pure illusions from the HVI and BI by a linear model, and found that these estimated values correlated significantly with the measured illusory effect in the variants of the illusions (pHVI and pBI). Our data show that illusory effects can be highly variable across participants, but are consistent in different versions of the illusions. In particular, the bisecting line can be perceived as either longer or shorter. These results show that it is important to analyze individual data to fully understand the underlying mechanisms of these illusions in the future.

\section{AUTHOR NOTE}

This research was supported by the German Science Foundation (Graduate Program "Neural Representation and Action ControlNeuroAct" 885/1). Parts of the results have been reported in abstract form (Hamburger, Hansen, \& Gegenfurtner, 2006). We thank Karl R. Gegenfurtner and Lothar Spillmann for valuable comments and discussions. Correspondence concerning this article may be addressed to K. Ham- 
burger, Justus-Liebig-Universität Giessen, Fachbereich 06, Abteilung Allgemeine Psychologie und Kognitionsforschung, Otto-BehaghelStrasse 10F, 35394 Giessen, Germany (e-mail: kai.hamburger@psychol .uni-giessen.de).

\section{REFERENCES}

ARMSTRONG, L., \& MARKs, L. E. (1997). Differential effects of stimulus context on perceived length: Implications for the horizontal-vertical illusion. Perception \& Psychophysics, 59, 1200-1213.

Avery, G. C., \& DAY, R. H. (1969). Basis of the horizontal-vertical illusion. Journal of Experimental Psychology, 81, 376-380.

Brainard, D. H. (1997). The Psychophysics Toolbox. Spatial Vision, 10, 433-436. doi:10.1163/156856897X00357

Brosvic, G. M., \& Cohen, B. D. (1988). The horizontal-vertical illusion and knowledge of results. Perceptual \& Motor Skills, 67, 463469. doi:10.2466/PMS.67.6.463-469

Chapanis, A., \& Mankin, D. A. (1967). The vertical-horizontal illusion in a visually-rich environment. Perception \& Psychophysics, 2 , 249-255.

DAY, R., \& AVERY, G. (1970). Absence of the horizontal-vertical illusion in haptic space. Journal of Experimental Psychology, 83, 172-173.

DeregowsKi, J., \& Ellis, H. D. (1972). Effect of stimulus orientation upon haptic perception of the horizontal-vertical illusion. Journal of Experimental Psychology, 95, 14-19.

Ebbinghaus, H. (1913). Grundzüge der Physiologie 2 (Vols. 1-3). Auflage: Leipzig.

Felleman, D. J., \& VAN Essen, D. C. (1991). Distributed hierarchical processing in the primate cerebral cortex. Cerebral Cortex, 1, 1-47. doi:10.1093/cercor/1.1.1-a

FICK, A. (1851). De errone quodam optic asymmetria bulbi effecto. Marburg: Koch.

FICK, A. (1852). Erörterung eines physiologisch-optischen Phänomens. Zeitschrift für rationelle Medicin, 2, 83-94.

FINGER, F. W., \& SPELT, D. K. (1947). The illustration of the horizontalvertical illusion. Journal of Experimental Psychology, 37, 243-250.

GARDNER, R. W., \& LONG, R. I. (1960a). Errors of the standard and illusion effects with the inverted T. Perceptual \& Motor Skills, 10, 47-54. doi:10.2466/PMS.10.1.47-54

Gardner, R. W., \& Long, R. I. (1960b). Errors of the standard and illusion effects with L-shaped figures. Perceptual \& Motor Skills, 10, 107-109. doi:10.2466/PMS.10.2.107-109

Gentaz, E., \& Hatwell, Y. (2004). Geometrical haptic illusions: The role of exploration in the Müller-Lyer, vertical-horizontal, and Delboeuf illusions. Psychonomic Bulletin \& Review, 11, 31-40.

Gregory, R. L. (1968). Perceptual illusions and brain models. Proceedings of the Royal Society B, 171, 279-296.

GRegory, R. L. (1997a). Knowledge in perception and illusion. Philosophical Transactions of the Royal Society B, 352, 1121-1128.

GREGORY, R. L. (1997b). Visual illusions classified. Trends in Cognitive Sciences, 1, 190-194. doi:10.1016/S1364-6613(97)01060-7

Gregory, R. L. (2001). Perceptions of knowledge. Nature, 410, 21. doi: $10.1038 / 35065174$

Hamburger, K., Hansen, T., \& Gegenfurtner, K. R. (2006). Geometric-optical illusions at isoluminance. In H. H. Bülthoff, S. Gillner, H. A. Mallot, \& R. Ulrich (Eds.), Beiträge zur 9. Tübinger Wahrnehmungskonferenz (p. 135). Kirchentellinsfurt: Knirsch.

Hamburger, K., Hansen, T., \& Gegenfurtner, K. R. (2007). Geometric-optical illusions at isoluminance. Vision Research, 47, 32763285. doi:10.1016/j.visres.2007.09.004
Heller, M. A., Calcaterra, J. A., Burson, L. L., \& Green, S. L. (1997). The tactual horizontal-vertical illusion depends on radial motion of the entire arm. Perception \& Psychophysics, 59, 1297-1311.

Heller, M. A., \& Joyner, T. D. (1993). Mechanisms in the haptic horizontal-vertical illusion: Evidence from sighted and blind subjects. Perception \& Psychophysics, 53, 422-428.

KoffKa, K. (1999). Principles of Gestalt psychology. London: Routledge. (Original work published 1935)

KÜNNAPAS, T. M. (1955). An analysis of the "vertical-horizontal" illusion. Journal of Experimental Psychology, 49, 134-140.

KünNAPAS, T. M. (1957). Vertical-horizontal illusion and surrounding field. Acta Psychologica, 13, 35-42. doi:10.1016/0001-6918 (57)90004-5

KüNNAPAS, T. M. (1958). Fixation and the vertical-horizontal illusion. Acta Psychologica, 14, 131-136. doi:10.1016/0001-6918 (58)90010-6

KÜNNAPAS, T. M. (1959). The vertical-horizontal illusion in artificial visual fields. Journal of Psychology, 47, 41-48.

McBride, S. A., Risser, J. M., \& Slotnick, B. M. (1987). The horizontal-vertical illusion: Independence of line bisection and a comparison line. Perceptual \& Motor Skills, 64, 943-948. doi:10.2466/ PMS.64.3.943-948

Millar, S., \& Al-Attar, Z. (2000). Vertical and bisection bias in active touch. Perception, 29, 481-500. doi:10.1068/p2989

OpPEL, J. J. (1855). Über geometrisch-optische Täuschungen. Jahresbericht des physikalischen Vereins zu Frankfurt am Main, 37-47.

PAN, S. (1934). The vertical-horizontal illusion. National Central Journal of Psychology, National Central University, 1, 125-128.

PelLi, D. G. (1997). The VideoToolbox software for visual psychophysics: Transforming numbers into movies. Spatial Vision, 10, 437-442. doi:10.1163/156856897X00366

Piaget, J., Matalon, B., \& Bang, V. (1961). L'evolution de l'illusion dite "verticale-horizontale" de ses composantes (rectangle et équerre) et de l'illusion de Delboeuf en presentation tachistoscopique. Archives de Psychologie, 38, 23-68.

Prinzmetal, W., \& Gettleman, L. (1993). Vertical-horizontal illusion: One eye is better than two. Perception \& Psychophysics, 53, 81-88.

RaudSEPP, J., \& DJupsJöBACKA, M. (2005). Handgrip maximum force and the visual horizontal - vertical illusion. Perception, 34, 421-428. doi:10.1068/p5073

RocKLAND, K. S., \& PANDYA, D. N. (1979). Laminar origins and terminations of cortical connections of the occipital lobe in the rhesus monkey. Brain Research, 179, 3-20. doi:10.1016/0006-8993(79)90485-2

Schumann, F. (1900). Beiträge zur Analyse der Gesichtswahrnehmungen. Zeitschrift für Psychologie \& Physiologie der Sinnesorgane, 24, $1-33$

Sillito, A. (1995). Chemical soup: Where and how drugs may influence visual perception. In R. L. Gregory, J. Harris, P. Heard, \& D. Rose (Eds.), The artful eye (pp. 294-306). Oxford: Oxford University Press.

vaN EsSEN, D. C. (1985). Functional organization of primate visual cortex. In A. Peters \& E. G. Jones (Eds.), Cerebral cortex (Vol. 3, pp. 259329). New York: Plenum.

Westheimer, G. (2008). Illusions in the spatial sense of the eye: Geometrical-optical illusions and the neural representation of space. Vision Research, 48, 2128-2142. doi:10.1016/j.visres.2008.05.016

(Manuscript received September 9, 2009; revision accepted for publication January 31, 2010.) 